\title{
A new promoter element associated with daily time keeping in Drosophila
}

\author{
Brandi Sharp ${ }^{1}$, Eric Paquet ${ }^{2}$, Felix Naef ${ }^{2}$, Akanksha Bafna ${ }^{3}$ and Herman Wijnen ${ }^{1,3, *}$ \\ ${ }^{1}$ Department of Biology, University of Virginia, Charlottesville, VA 22903, USA, ${ }^{2}$ Institute of Bioengineering, School of \\ Life Sciences, Ecole Polytechnique Fédérale de Lausanne, Lausanne, Switzerland and ${ }^{3}$ Biological Sciences and \\ Institute for Life Sciences, University of Southampton, Southampton SO17 1BJ, UK
}

Received November 07, 2016; Revised March 06, 2017; Editorial Decision March 27, 2017; Accepted April 11, 2017

\begin{abstract}
Circadian clocks are autonomous daily timekeeping mechanisms that allow organisms to adapt to environmental rhythms as well as temporally organize biological functions. Clock-controlled timekeeping involves extensive regulation of rhythmic gene expression. To date, relatively few clock-associated promoter elements have been identified and characterized. In an unbiased search of core clock gene promoters from 12 species of Drosophila, we discovered a 29-bp consensus sequence that we designated as the Clock-Associated Transcriptional Activation Cassette or 'CATAC'. To experimentally address the spatiotemporal expression information associated with this element, we generated constructs with four separate native CATAC elements upstream of a basal promoter driving expression of either the yeast Gal4 or firefly luciferase reporter genes. Reporter assays showed that presence of wild-type, but not mutated CATAC elements, imparted increased expression levels as well as rhythmic regulation. Part of the CATAC consensus sequence resembles the E-box binding site for the core circadian transcription factor CLOCK/CYCLE (CLK/CYC), and CATACmediated expression rhythms are lost in the presence of null mutations in either cyc or the gene encoding the CLK/CYC inhibitor, period (per). Nevertheless, our results indicate that CATAC's enhancer function persists in the absence of CLK/CYC. Thus, CATAC represents a novel cis-regulatory element encoding clock-controlled regulation.
\end{abstract}

\section{INTRODUCTION}

The circadian clock of higher eukaryotes is understood to be a conserved transcription/translation auto-regulatory feedback mechanism controlled via rhythmic transcriptional activation and repression. Mammals as well as in- sects have circadian clocks that operate via interlocking transcriptional feedback loops that rely on heterodimers of basic helix-loop-helix (bHLH), Per-Arnt-Sim (PAS) domain transcription factors (TFs) for transcriptional activation (1). In the clock circuit of the fruit fly, Drosophila melanogaster, a heterodimer consisting of CLOCK (CLK) and CYCLE (CYC), acts as the core transcription factor $(2,3)$. CLK/CYC binds DNA by associating with a canonical CACGTG E-box or E-box-like sequences (4-6). The detection of transcripts regulated by CLK/CYC is of particular interest to the field of circadian biology. E-boxes, however, are poor predictors of potential clock-regulated genes because the sequence motif is widespread throughout the fly genome. The nucleotides flanking the E-box and/or an arrangement of closely spaced associated motifs, on the other hand, likely contribute to clock transcriptional activity, TF specificity and increased binding affinity $(7,8)$.

In Drosophila, studies of the promoter regions of known clock genes, period (per) and timeless (tim), found that Ebox-dependent enhancers are necessary for circadian transcriptional modulation. The per promoter has, arguably, the best studied circadian enhancer motif to date. The enhancer is a 69-bp sequence upstream of the transcription start site (TSS) that activates circadian gene expression (5). This enhancer relies on an E-box motif to mediate transcriptional activation and $3^{\prime}$ sequences near the E-box to drive strong amplitudes and tissue specific expression $(5,9)$. However, the core of the 69-bp enhancer is actually an E-box-bearing 18-bp region that is able, when multimerized, to recapitulate per spatial and temporal expression (4). The tim promoter possesses closely spaced E- and TER ( $\operatorname{tim}$ E-box-like repeats) boxes, which are a variant of the consensus E-box sequence (6). This tim enhancer relies on two non-canonical E-boxes, TER 1 and TER2, to strongly initiate gene expression and cycling amplitude. The canonical E-box present in the enhancer appears to be non-functional on its own and seems to require the TER boxes to elicit functionality.

Increasing knowledge of the Drosophila genome, annotated and cloned full-length cDNAs, transcription start sites (TSSs) and transcription factor binding sites (TFBSs) have provided more tools to determine the elements re-

\footnotetext{
${ }^{*}$ To whom correspondence should be addressed. Tel: +44 23 80594336; Fax: +44 23 8059 5159; Email: hw3a11@soton.ac.uk
} 
sponsible for transcriptional regulation $(10,11)$. In previous work (12), we identified a conserved motif consisting of two closely spaced E-box-like elements in the 69-bp per enhancer as well as in other CLK/CYC-controlled genes (tim, vrille, Par domain protein 1 and clockwork orange). As a continuation of this work, we, hereby, identified a second novel and independent 29-bp motif. The present study describes spatiotemporal expression information contributed by the latter motif, which was named Clock-Associated

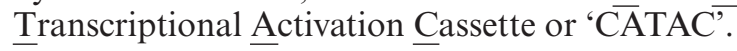

\section{MATERIALS AND METHODS}

\section{Identification of CATAC consensus sequence}

The bioinformatics model used to predict the 'CATAC' element is identical to that previously described for the discovery of the E1-E2 element (12). Briefly, we used MEME (13) to scan the genomic sequences in windows of $2.5 \mathrm{~kb}$ around the TSSs of tim, vrille, Pdpl and cwo (stichl) in 12 Drosophila species (MultiZ alignments were downloaded from UCSC, dm3 assembly). This identified a conserved set of CATAC sequences (Supplementary Figure S1). In order to obtain our final position weight matrix (Figure 1A), we trained a Hidden Markov Model as in (12), but consisting only of a single 29 bp motif, using the MEME-derived consensus as a seed. A .bed file that identifies all CATAC sites with a bit score $>5$ in the Drosophila melanogaster genome (BDGP R5/dm3) is included in the supplemental information.

\section{Generating CATAC reporter constructs}

Synthetic oligonucleotides were designed to match four separate, native occurrences of the CATAC element from either the Pdp1 or Slob promoters: Slob 4xCATAC enhancer, 5'pGGCCGATAACGCGGCGTATGCGCAA TGTCGAAGCATATTACGCATACGCCCCATCCGC; 5'pGTTTGCTGTGGCGGATGGGGCGTATGCGTAA TATGCTTCGACATTGCGCATACGCCGCGTTATC; 5'pCACAGCAAACGCTGCGTATGCGTAATACTTT GTGCACACGTTGCGTATGAGTAATGTCCT; 5'pA ATTAGGACATTACTCATACGCAACGTGTGCAC AAAGTATTACGCATACGCAGC, Slob mt4xCATAC enhancer, 5'pGGCCGATATCCCGGCCTTTGCCCTA TGTCGAAGCATATAAGGCAAGGCCCGAACCGC; 5'pGATTGCTGTGGCGGTTCGGGCCTTTGCCTTA TATGCTTCGACATAGGGCAAAGGCCGGGATATC; 5'pCACAGCAATCCCTGCCTTTGCCT TTGTGCACTCCTTGCCTTTGACTTATGTCCT; 5'pAATTAGGACATAAGTCAAAGGCAAGGAGT GCACAAAGTATAAGGCAAAGGCAGG, $P d p 1$ 4xCATAC enhancer, 5'pGGCCAGCACATTACGCA TACGTCACGTGTTGCAAAAATAATACGTATAC GACGCGTGTC; 5'pTGGTACAGAAGACACGC GTCGTATACGTATTATTTTTGCAACACGTGAC GTATGCGTAATGTGCT; 5'pTTCTGTACCATGC GGCGTATGAGCAATCTGTTAATACGTTACC CATACGCCCCGTGGGC C; 5'pAATTGGCCCAC GGGGCGTATGGGTAACGTATTAACAGATTG CTCATACGCCGCA, Pdpl mt4xCATAC enhancer, 5'pGGCCAGCACATAAGGCAAAGGTCAGGAGT
TGCAAAAATAAAAGGTAAAGGACGGGAGTC; 5'pAGGTACAGAAGACTCCCGTCCTTTACCTTTT ATTTTTGCAACTCCTGACCTTTGCCTTATGTGCT; 5'pTTCTGTACCTTC CGGCCTTTGACCTATCTG TTAATACGTA AGCCAAAGGCCCGGAGGGCC; 5'pA ATTGGCCCTCCGGGCCTTTGGCTTAC GTATTAACAGATAGGTCAAAGGCCGGA,

Pdp1 4xCATAC with Slob-like E-boxes enhancer, 5'pGGCCAGCACATTACGCATACGTAACGTGT TGCAAAAATAATACGTATACGAAGCGTTTC; 5'pTCGTACAGA AGAAACGCTTCGTATACGTA TTATTTTTGCAACACGTTACGTATGCGTAATGT GCT; 5'pTTCTGTACGATGGGGCGTATGAGCAAT CTGTTAATACGTTACCCATACGCCGCGTTGGC C; 5'pAATTGGCCAACGCGGCGTATGGGTAACGT ATTAACAG ATTGCTCATACGCCCCA. Oligos were annealed to match their order and orientation in the native Pdpl or Slob promoters. Flanking EagI and EcoRI sequences allowed for the 4xCATAC enhancers to be inserted upstream of the $h s p 70$ basal promoter in the pPTGAL vector (14) (obtained from the Drosophila Genomics Resource Center). The luciferase constructs (pPTluc) were created by blunt-end cloning to replace a $3193 \mathrm{bp}$ fragment containing Gal4 flanked by PstI sites with a 1955 bp HindIII-BamHI fragment containing the luciferase gene from pGL3-Basic (Promega) (15). Copies of pPTluc can be requested online (http://www.addgene.org/Herman_Wijnen/). For each reporter construct, several independent lines were generated.

\section{Drosophila stocks}

Fly stocks bearing the $w^{1118}, U A S-C D 8: \because G F P$, and eya $a^{2}$ mutations were obtained from the Bloomington Drosophila Stock Center. The $c y c^{01}, p e r^{01}$ and tim-luc alleles have been described previously $(3,16,17)$. All stocks were maintained on standard yeast cornmeal agar food.

\section{In vivo luciferase monitoring}

Bioluminescence monitoring of flies was carried out as described previously (17-19). $100 \mu \mathrm{l}$ of a $5 \%$ sucrose $1 \%$ agar solution containing $15 \mathrm{mM}$ luciferin (GOLDBIO) was added to every other well of a white 96-well microtiter plate (Optiplate, Perkin Elmer). Flies were entrained for 3 days in a 12-h light, 12-h dark cycle (LD 12:12). On day 3 during the light phase, flies were anesthetized, added to separate wells and covered with clear plastic domes to reduce noise caused by fly movement closer to and farther away from the photodetector. Plates were then placed into a TopCount Scintillation Counter and subjected to the remaining portion of the LD phase and subsequent constant darkness (DD). Luminescence from each fly was monitored for 7-17 s per time point, thereby allowing data for each fly to be collected roughly once every hour.

\section{Quantitative data analysis}

Luciferase assay data were analyzed for period in the circadian range (15-35 h) and relative amplitude error (RAE) by an iterative, coupled fast Fourier transform-non-linear 
A

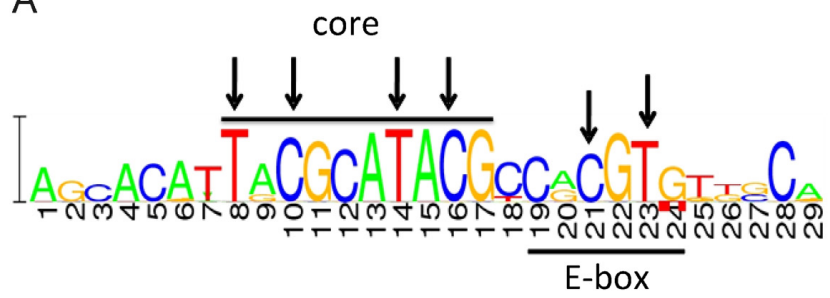

B
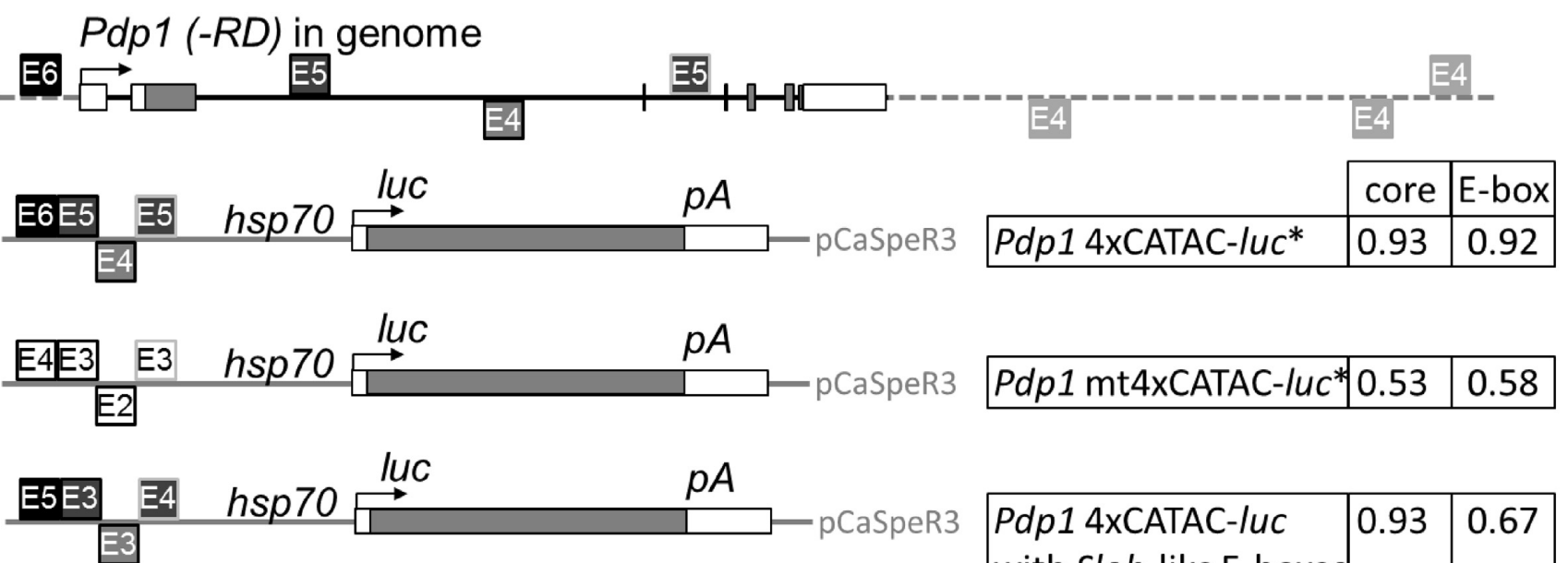

\begin{tabular}{|l|l|l|}
\hline $\begin{array}{l}\text { Pdp1 4xCATAC-luc } \\
\text { with Slob-like E-boxes }\end{array}$ & 0.93 & 0.67 \\
\hline
\end{tabular}

Slob $(-R B)$ in genome
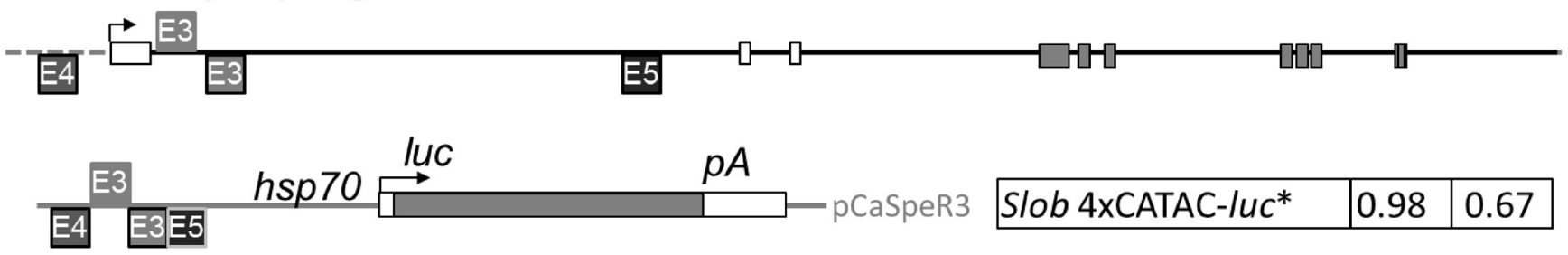

\begin{tabular}{|c|c|c|c|c|c|}
\hline$F 1$ & $\underset{\longrightarrow}{\operatorname{lu} C}$ & $p A$ & & & \\
\hline$E 2$ & $n s p / 0$ & - pCaSpeR3 & Slob mt4xCATAC-Iuc* & 0.58 & 0.33 \\
\hline E5E3 & 20 & $p A$ & & & \\
\hline ESE- & nsp/u & _- pCaSpeR3 & $\begin{array}{l}\text { Slob 4xCATAC-luc } \\
\text { Inverted enhancer }\end{array}$ & 0.98 & 0.67 \\
\hline
\end{tabular}

Figure 1. Clock-associated transcriptional activation cassette (CATAC) and reporter constructs. (A) Consensus sequence for the CATAC element derived from five clock genes (tim, per, cwo, vri and $P d p 1$ ) from 12 Drosophila species. The highly conserved core region is overlined and the E-box sequence match is underlined. Residues mutated in this study are marked with arrows. (B) CATAC-luciferase reporter constructs used in this research. Reporters were generated by inserting CATAC sequences from the Pdpl or Slob genes upstream of the basal promoter in the pPTluc vector. Individual CATAC elements are indicated as E1/E2/E3/E4/E5/E6 depending on the match of their E-box region to the CACGTG consensus (1-6 matching residues). The native CATAC elements used for the $P d p 1$ and Slob CATAC constructs are also shown in their genomic context with the $P d p 1-R D$ and $S l o b-R B$ transcripts, respectively (the patterns of shading and outline color allow matching between transgenic elements and their genomic origin). The mt4xCATAC constructs contain six $(\mathrm{A} \Leftrightarrow \mathrm{T}$ or $\mathrm{C} \Leftrightarrow \mathrm{G})$ transversion mutations in each of the CATAC elements at the positions indicated in (A). 'Pdp1 4xCATAC-luc with Slob-like E-boxes' (PSE) was created by changing the E-box consensus matches of the original elements as indicated. The 'core' and 'E-box' scores for each construct represent the fraction of residues in each of the four elements matching the respective TRCGCATACG and CRCGTG consensus sequences from (A). For constructs marked with an asterisk, additional versions carrying Gal4 instead of luc as the reporter were generated. Sequences are specified in Materials and Methods. 
least squares (FFT-NLLS) multicomponent cosine analysis (19). RAE is the ratio of the $95 \%$ confidence interval by the amplitude estimate - the ratio of amplitude error to most probable amplitude. Lower RAE values suggest stronger rhythms. In our analysis, individual flies with RAE values $<0.7$ were considered rhythmic, $\geq 0.7$ were considered weakly rhythmic, and those for which the program returned no data (RAE > 1), arrhythmic. The first 24-h of data were excluded from the plots and quantitative analysis since flies placed on fresh luciferin media require time to inactivate previously synthesized luciferase. For each fly, only DD data were analyzed for statistical analysis.

\section{Imaging of GFP and luciferase reporters}

Larvae and adult flies producing 4xCATAC-Gal4 driven expression of membrane-tethered green fluorescent protein were dissected in Ringer's solution (20) under a dissecting scope equipped with UV light. Tissues expressing GFP were wet or dry mounted and immediately imaged with a fluorescence microscope equipped with a CCD camera. Luciferase expressing flies were dehydrated for $24-\mathrm{h}$ then fed on a cotton plug soaked in $200 \mu \mathrm{l}$ of a $1 \%$ sucrose solution containing $15 \mathrm{mM}$ luciferin (GOLDBIO). Feeding occurred for $1-2 \mathrm{~h}$ prior to imaging of male heads and for 25-26 h prior to imaging of male bodies. Luciferaseexpressing adult fly heads or bodies were mounted on a sterile filter insert (Millicell-CM; Millipore Inc.) and immobilized under a 13-mm coverglass with the help of sterile vacuum grease (21). The insert was then placed in a sterile glass-bottom dish (FluoroDish FD35PDL) containing insect tissue culture media with $0.1 \mathrm{mM}$ luciferin. Sterile vacuum grease was then applied to seal the dish. Luminescence imaging was conducted as previously described by Sellix et al. (21), with an inverted epifluorescence microscope (Olympus CKX-41 equipped with a cooled intensified CCD camera (Mega10Z; Stanford Photonics Inc., Palo Alto, CA, USA) housed in a light-tight wooden dark box. Luminescence images were collected using Piper image analysis software (Stanford Photonics).

\section{Quantitative reverse transcriptase PCR (qRT-PCR) analyses}

qRT-PCR expression analyses were carried out as described previously $(22,23)$. Slob or Pdp1 4xCATAC-luciferase flies were entrained to $12 \mathrm{~h}$ light $/ 12 \mathrm{~h}$ dark cycles at $25^{\circ} \mathrm{C}$ prior to release into constant conditions (DD $25^{\circ} \mathrm{C}$ ). Flies were harvested onto dry ice at time points CT0, CT6, CT12, CT18 and CT24, and adult heads were dissected on a chilled platform and transferred to guanidinium thiocyanate buffer. Four separate groups of flies ( $\sim 50$ each) were used for each experimental condition. Total RNA was obtained from the heads using the RNAqueous4PCR kit (Ambion). Sample concentration and purity was analysed using a NanoDrop spectrophotometer. Samples exhibited OD 260/280 ratios between 1.8 and 2.1. Concentrations were adjusted to 25 $\mathrm{ng} / \mu \mathrm{l}$ in $10 \mathrm{mM}$ Tris-HCl $0.1 \mathrm{mM}$ EDTA $\mathrm{pH} 8.0$ buffer and samples were frozen at $-80^{\circ} \mathrm{C}$ in aliquots until further use. The RNA samples were then analyzed with the SensiFAST SYBR No-ROX One-Step qPCR Kit (Bioline) using experimental primer pairs designed to specifically amplify fragments of the circadian Pdp1 or Slob transcripts, the transgenic luciferase transcript or the EFI $\beta$ control transcript (see Supplementary Table S1). All primers and amplicons used in this study have been described before $(22,23)$. Oligonucleotides were sourced from Integrated DNA Technologies ( $25 \mathrm{nmol}$ scale, standard desalting purification). No-template and RNAse-treated controls were included to avoid false positive results. The following thermocycling protocol was used for the $P d p 1$ and Slob amplicons: $60^{\circ} \mathrm{C}$ $180 \mathrm{~s}, 95^{\circ} \mathrm{C} 300 \mathrm{~s},\left[95^{\circ} \mathrm{C} 15 \mathrm{~s}, 62^{\circ} \mathrm{C} 30 \mathrm{~s}\right] \times 45,40^{\circ} \mathrm{C} 60 \mathrm{~s}$, melting curve $60^{\circ} \mathrm{C}$ to $95^{\circ} \mathrm{C} 0.2^{\circ} \mathrm{C} / \mathrm{s}$. Thermocycling for $l u$ ciferase and EFI $\beta$ made use of an adjusted annealing temperature of $60^{\circ} \mathrm{C}$ instead of $62^{\circ} \mathrm{C}$ during the 45 amplification phase. Expression levels measured on a SmartCycler system (Cepheid) relative to $E F 1 \beta$ were determined using the comparative Cycle threshold (Ct) method (24) and analysis of timed gene expression was carried out after normalization to the time course average. Amplicon sizes and specificity were verified by $2 \%$ agarose gel electrophoresis of all experimental and control PCR samples. Statistical analyses were conducted using SPSS.

\section{RESULTS}

\section{Identification of a conserved promoter element in Drosophila}

In order to detect over-represented cis-acting elements associated with clock gene expression, we combined MEME (13) with a Hidden Markov Model ((12) and Materials and Methods) to analyse an alignment of promoter sequences of the core clock genes (per, tim, Pdp1, cwo and vri) for 12 species of Drosophila. As a result, a 29-bp motif designated the Clock-Associated Transcriptional Activation Cassette or 'CATAC', was identified as over-represented in these promoters (Figure 1A, Supplementary Figure S1). The consensus bears a well-conserved core' motif (nucleotides 8-17) and, although the model was not seeded with an E-box, it bears an E-box-like motif as well (nucleotides 19-24) (Figure 1A). To our knowledge, no functional analyses of individual CATAC elements or corresponding genomic sites have been reported to date. An illustration of the relationship between the CATAC element and the major known circadian enhancer element in the per gene (69-bp enhancer (5)) is provided in supplemental Supplementary Figure S1B.

A possible association of CATAC with clock-controlled and/or circadian transcription was explored further by determining the relative frequencies at which the CATAC element occurred in relevant promoter sequences outside of the five training genes. In particular, enrichment of CATAC was found in the top $0.5 \%$ genes $(n=61$ genes) predicted to be induced upon activation of the circadian regulator CLK (25) as well as in the top $0.7 \%$ genes $(n=97$ genes) encoding transcripts predicted to exhibit circadian oscillations (25-28) (Supplementary Figure S2). A prominent circadian transcript associated with CATAC promoter elements is Slob (Slowpoke binding protein) (27). With the exception of the element in the first intron of per, all CATAC elements associated with these six genes coincide with conserved sequence elements predicted by PhastCons analysis from an alignment of 27 insect genomes (29).

Based upon the sequence alignment, conservation, and subsequent CLK/CYC target gene enrichment analyses, 
A
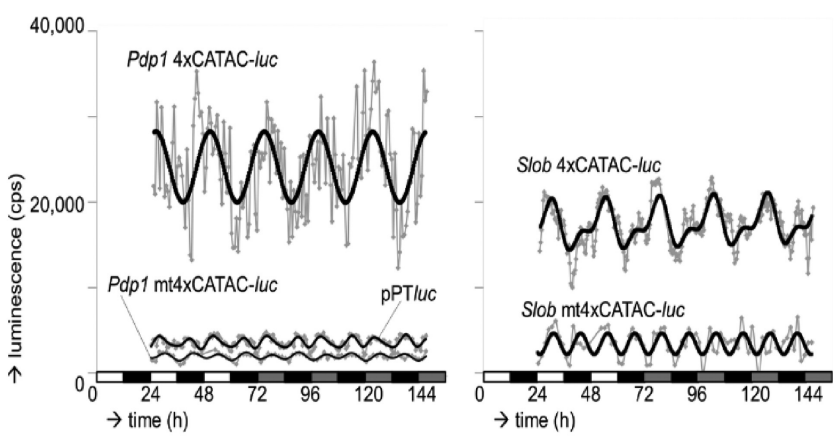

B

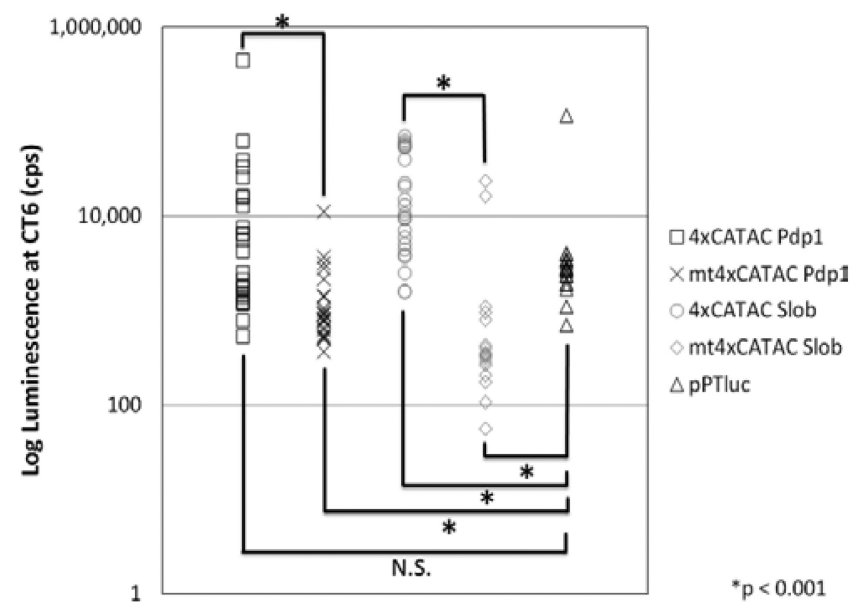

C

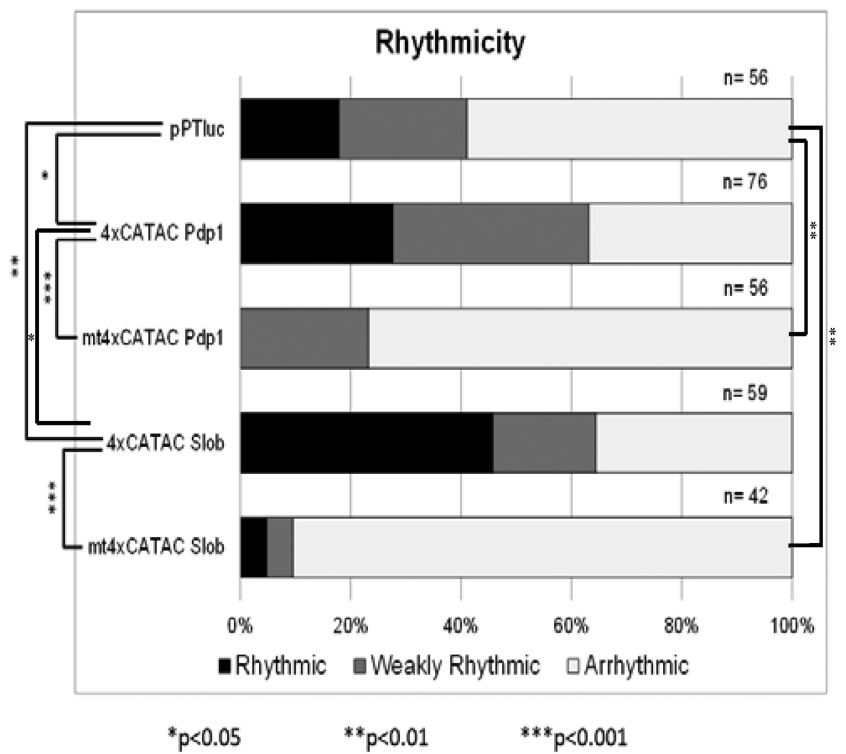

Figure 2. Regulation of 4xCATAC-luc reporter constructs. (A) Comparison of detrended, average traces (gray) and their corresponding FFT theoretical overlays (black) demonstrated a strong rhythm in wild-type CATAC-luc reporter activity. Average traces were derived from $26 P d p 1$ 4xCATAC-luc, 23 Pdpl mt4xCATAC-luc and 19 pPT luc lines (left panel) as well as 23 Slob 4xCATAC-luc and 17 Slob mt4xCATAC-luc lines (right panel). The luminescence profiles of reporters with mutant CATAC elements showed weaker, ultradian rhythms that resembled those observed
CATAC was hypothesized to be a regulatory element involved in mediating spatiotemporal expression of clockregulated genes.

\section{CATAC imparts rhythmicity to a luciferase reporter}

To test our hypothesis, we studied CATAC elements found in two genes, Pdpl and Slob, that not only exhibit strong CLK/CYC-associated circadian oscillations, but also have an unusually high number of CATAC motifs. The $P d p 1$ gene encodes a clock component (PDP1- $\epsilon$ ) that impacts molecular circadian oscillations, particularly in the clock neurons, as well as circadian behaviour $(30,31)$. Remarkably, Pdpl has seven CATAC elements in close proximity, one of which bears a canonical E-box (Figure 1B). The Slob gene, which encodes proteins that regulate the slowpoke channel, exhibits strong circadian regulation of its expression. The Slob promoter has four CATAC elements which all possess noncanonical E-boxes. To address the spatiotemporal expression information associated with CATAC, enhancer elements were generated, bearing four separate native CATAC elements from the Slob or Pdpl promoters (Figure 1B; Supplementary Figure S3). Each enhancer was assembled such that the four CATAC elements matched their order and orientation in the native $P d p 1$ and Slob promoters (Figure 1B; Supplementary Figure S3). Moreover, like their promoters, the Pdpl 4xCATAC enhancer possesses one CATAC element with a canonical E-box while the Slob 4xCATAC enhancer has four noncanonical E-boxbearing CATAC elements. More specifically, in comparison with the Pdp1 4xCATAC enhancer the Slob 4xCATAC enhancer has more mismatches relative to both the CACGTG canonical E-box consensus (9/24 versus 4/24 mismatches) as well as the CRCGTG consensus for the E-box-like element within CATAC (Figure 1A; 8/24 versus 2/24 mismatches).

Mutated CATAC constructs were also generated by making transversion mutations to each of the four native elements. Mutations were made to the well-conserved core motif at residues 8, 10,14 and 16, as well as to the E-box-like motif at residues 21 and 23 (Figure 1A; Supplementary Figure S3). Native and mutated 4xCATAC enhancers were then inserted upstream of a basal promoter driving expression of the firefly luciferase reporter gene (Figure 1B). Multiple independent transgenic lines were generated by $\mathrm{P}$ element

for the enhancer-less control (pPTluc). (B) 4xCATAC-induced reporter expression was determined after $6 \mathrm{~h}$ in DD from non-detrended, averaged raw data. The expression levels of $P d p 1$ and Slob 4xCATAC were higher than their mutant counterparts $(P<0.001$, Mann-Whitney U/Wilcoxon $\mathrm{W}$ rank sum test). The pPTluc expression levels were significantly higher than Pdpl and Slob mt4xCATAC but lower than Slob 4xCATAC. (C) 4xCATAC-luc reporter rhythmicity was determined from TopCount in vivo luminescence data by the FFT-NLLS utility of the BRASS software program. Individual flies with relative amplitude error (RAE) $<0.7$, between 0.7 and 1 , or $>1$ were classified as rhythmic, weakly rhythmic and arrhythmic, respectively. Pairwise Chi-square test comparisons were used to generate the indicated $P$ value estimates. $P d p 1$ and Slob 4xCATAC generated greater rhythmicity than their mutated counterparts. Residual rhythmicity was observed for empty pPTluc vector, and to a lesser degree in mutant $4 \times C A T A C$ constructs, but this was always significantly reduced relative to the wild-type $P d p 1$ and Slob 4 xCATAC reporters. 
Table 1. Comparison of in vivo luminescence rhythms of different $4 \times$ CATAC-luc reporters

\begin{tabular}{|c|c|c|c|c|c|c|c|}
\hline Genotype & \#lines & \#flies & $\% \mathrm{R}$ & $\% \mathrm{WR}$ & $\% \mathrm{AR}$ & $\begin{array}{l}\text { Mean RAE } \pm \\
\text { SEM }\end{array}$ & $\begin{array}{l}\text { Mean period } \pm \\
\text { SEM }\end{array}$ \\
\hline Pdpl 4xCATAC-luc & 26 & 76 & 28 & 36 & 37 & $0.69 \pm 0.02^{\mathrm{a}, \mathrm{b}}$ & $24.09 \pm 0.26^{\mathrm{g}}$ \\
\hline$P d p 1$ with Slob-like E-boxes 4xCATAC-luc & 18 & 105 & 44 & 25 & 31 & $0.62 \pm 0.02^{\mathrm{c}}$ & $23.01 \pm 0.30$ \\
\hline Slob 4xCATAC-luc & 23 & 59 & 46 & 19 & 36 & $0.57 \pm 0.02^{\mathrm{b}, \mathrm{f}}$ & $24.72 \pm 0.53^{\mathrm{h}}$ \\
\hline Flipped Slob 4xCATAC-luc & 8 & 71 & 32 & 31 & 37 & $0.68 \pm 0.03^{\mathrm{d}}$ & $23.17 \pm 0.53$ \\
\hline Slob mt4xCATAC-luc & 17 & 42 & 5 & 5 & 90 & $0.71 \pm 0.07$ & $24.15 \pm 0.83$ \\
\hline
\end{tabular}

RAE: Relative Amplitude Error, only provided for weakly rhythmic and rhythmic flies. \%R/WR/AR: percentage of single fly luminescence traces that are rhythmic, weakly rhythmic or arrhythmic.

ANOVA with post-hoc Tamhane's T2 $P<0.001^{\mathrm{a}, \mathrm{d}, \mathrm{e}} ; P<0.01^{\mathrm{b}, \mathrm{c}, \mathrm{g}, \mathrm{h}} ; P<0.05^{\mathrm{f}}$.

transformation of native and mutated 4xCATAC Pdp1 or $S l o b$ reporter constructs. Transformants were tested in an automated bioluminescence assay (TopCount) which allowed measurement of the transcriptional reporter constructs at relatively high frequency in vivo. Native $P d p 1$ and Slob $4 \mathrm{xCATAC}$ both showed strong luciferase expression (Figure 2, Supplementary Figure S4) and rhythms (Figure 2, Supplementary Figure S5; Table 1), and their average profiles showed a single peak per $24 \mathrm{~h}$ (Figure 2). Thus, the presence of a canonical E-box, which was lacking from the Slob 4xCATAC reporter, did not seem to be required for either rhythms or induction. A similar conclusion was reached in a previous study of the tim promoter, which showed that noncanonical E-boxes can significantly contribute to circadian transcriptional activity (6). If anything, native $P d p 1$ 4xCATAC transgenes, which included better matches to both the canonical and CATAC E-box consensus sequences tended to exhibit somewhat lower levels of rhythmicity than native Slob 4xCATAC transgenes with poorer quality Eboxes (Figure 2, Supplementary Figure S5; Table 1).

When we mutated all elements in the Pdpl and Slob 4xCATAC enhancers, the luciferase expression levels decreased (Figure 2, Supplementary Figure S4) and the rhythms were disrupted (Figure 2, Supplementary Figure S5; Table 1). Thus, the CATAC consensus sequence contributes to reporter induction and rhythmicity. Empty vector (pPTluc) and both mt4xCATAC constructs shared characteristically low level expression (Figure 2,Supplementary Figure S4) and comparable oscillation patterns, with twopeaks occurring per 24-hrs (Figure 2, Supplementary Figure S6). Earlier studies also found this 12-hr rhythm component in mutant E-box and basal promoter controls (4) and it has been reported to be a component of all expression patterns in such bioluminescence assays $(4,19,32)$.

Although pPTluc and both of the mt4xCATAC constructs exhibited residual rhythmicity, neither exhibited the proportion of rhythmic flies observed for native $P d p 1$ or Slob 4xCATAC (Figure 2). The residual rhythm for these constructs may have arrisen due to cryptic regulatory element(s) present in the reporter vector or, alternatively, as a result of rhythmic post-transcriptional modulation of luciferase reporter activity in the tissues expressing these constructs. We also generated two additional constructs, 'flipped Slob' (FS) in which the orientation of Slob $4 \times C A T A C$ in the vector was inverted and 'Pdpl 4xCATAC with Slob-like E-boxes' (PSE) in which the four E-boxes of
Pdp1 4xCATAC were mutated such that their sequence fidelity matched that of the four E-boxes in Slob 4xCATAC (Figure 1B; Supplementary Figure S3). These constructs also exhibited better rhythmicity than pPTluc and both mt4xCATACs (Supplementary Figure S5; Table 1). Moreover, FS and PSE induced significantly more luciferase activity than the respective $S l o b$ and $P d p 1$ mt4xCATAC controls (Supplementary Figure S4). Thus, in sum, in vivo luciferase reporter analyses uncovered preferential activity induction and rhythmicity for constructs containing wildtype CATAC elements.

We performed ANOVA analyses of luminescence data using only rhythmic and weakly rhythmic individual flies as determined by FFT-NLLS. The coherence of rhythms observed for Pdpl and Slob 4xCATAC reporters, as measured by the inversely correlated Relative Amplitude Error (RAE), was significantly stronger than those for mt4xCATAC and empty vector controls, respectively (Table 1). Period measurements, did not differ significantly between reporter constructs, except for the Pdp1 4xCATAC versus Pdpl mt4xCATAC comparison (Table 1). However, the unexpected short period length observed for $P d p 1$ mt4xCATAC rhythms may be attributable to reduced accuracy in period estimation due to weak rhythms for this reporter.

\section{CATAC-driven reporters are expressed in the eye and other} tissues with peak transcript phases during (subjective) day

To further examine the spatiotemporal expression of the CATAC reporter, we crossed our 4xCATAC-Gal4 lines with $U A S-C D 8: \because G F P$ flies to generate offspring that report CATAC activity by expression of membrane-tethered green fluorescent protein. In larvae and adult flies, 4xCATAC expression primarily included the salivary glands in addition to the photoreceptor cells of the compound eye in the adult (Figure 3A, Supplementary Figure S7). We also examined brains of the 4xCATAC flies but found no evidence of GFP expression. Little is known about the salivary glands as a circadianly rhythmic tissue and, although the observed signal in salivary glands was not due to autofluorescence or leaky expression of the UAS-CD8::GFP transgene (Supplementary Figure S7B), it is possible that the reporter gene expression in this tissue reflects the presence of a suspected salivary gland enhancer in the $h s p 70$ sequences (33) included in the pPTGal vector. Conversely, the photorecep- 

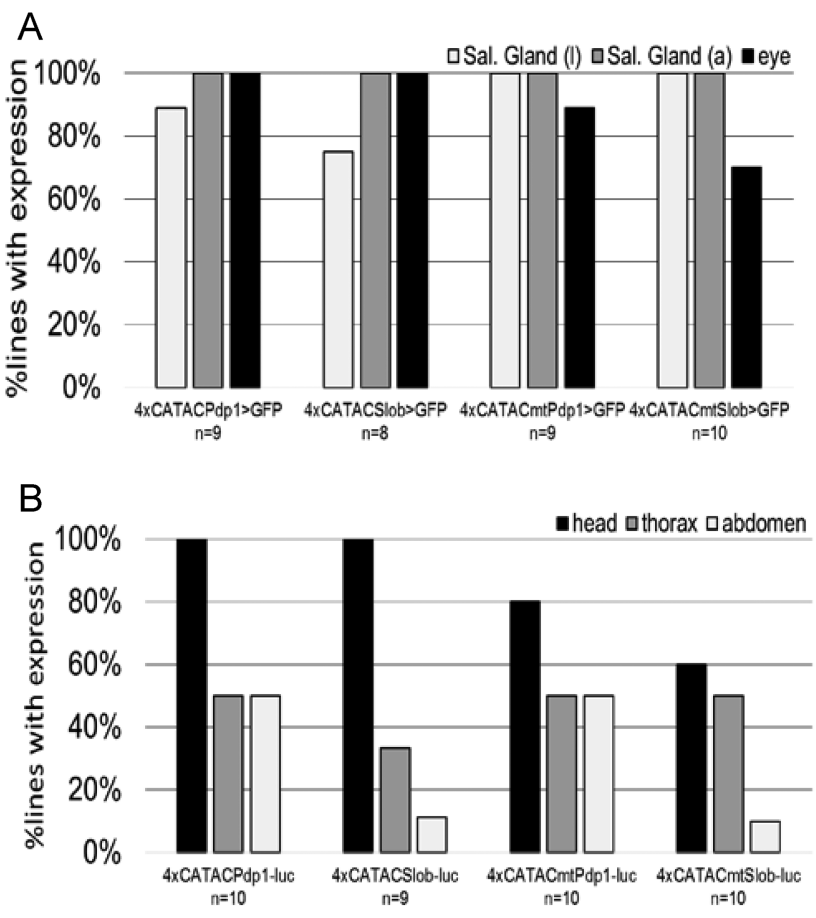

Figure 3. Spatial expression patterns of transgenic CATAC reporters. Larvae (1) and adult (a) male flies from multiple (n) lines expressing either 4xCATAC-Gal4-mediated GFP (A) or 4xCATAC-luciferase (B) were dissected to determine the spatial expression pattern of CATAC by fluorescence or luminescence imaging, respectively. The proportion of lines with tissues positive for a given reporter is indicated.

tors are known to possess an autonomous, circadian oscillator (34). Furthermore, both the native Slob and Pdpl transcripts are expressed in the photoreceptors $(30,35)$.

To follow-up, we imaged dissected heads and bodies of male Pdpl and Slob 4xCATAC luciferase flies that were fed a sucrose solution containing $15 \mathrm{mM}$ of luciferin, as in the TopCount assays. These tissues were then cultured in luciferin-containing media and imaged as close to their LD peak expression time as possible. The peak times were determined from the previously described TopCount assays (Figure 2) - Pdp1 (ZT 2-3) and Slob (ZT 3-5). Heads showed luciferase expression in not only the compound eyes, but also the proboscis and antennae; each of which has been shown to possess a circadian oscillator $(17,18)$ (Figure 3B, Supplementary Figure S8A). The bodies (including appendages) demonstrated signal in the wing, another tissue with an oscillator, and in other tissues within the thorax and abdomen (Figure 3B, Supplementary Figure S8B).

Given the status of the adult compound eye as a tissue with not only native expression of Pdpl and Slob $(30,35)$ but also $>80 \%$ of the circadian oscillators in fly heads (36), follow-up experiments were conducted to determine the contribution of the compound eyes to overall 4xCATAC reporter signal. We genetically removed the eyes via the eyes absent mutation, eya ${ }^{2}$ (37). While the luciferase expression levels in these flies did decrease, there was still significant luciferase activity (Supplementary Figure S9, Table 2). Thus, while the eyes are a source of $4 x$ CATAC driven luciferase signal, they are not its only relevant source.
Therefore, spatial expression analyses of the wild-type and mutant 4xCATAC reporters uncovered expression in a number of tissues including the known oscillators of the compound eye. However, despite some trends suggesting a role for eye clocks in CATAC-mediated rhythmicity, no statistically significant differences between the spatial expression patterns of wild-type and mutant 4xCATAC reporters were uncovered (Figure 3, Supplementary Figure $\mathrm{S} 8$ ). As described above, this stands in contrast to clear differences in rhythmicities (Figure 2, Supplementary Figure S5, Table 1) and expression levels (Figure 2, Supplementary Figure S4, Table 1) produced by wild-type versus mutant 4xCATAC-luc constructs. Taken together, these findings suggest that CATAC primarily specifies temporal rather than spatial information.

The native $P d p 1$ and $S l o b$ transcripts oscillate robustly in fly heads (25-28). While we had found that Pdpl and Slob 4xCATAC-luc oscillated in whole flies, it remained unclear whether the $4 \mathrm{xCATAC}$ reporter transcripts oscillated in the same phase as their respective native genes. To address this question, we performed a qRT-PCR time course analysis on native and reporter gene mRNA in whole fly heads. We found that both $P d p 1$ and Slob 4xCATAC-luc oscillated in phase with each other, peaking at CT6 (Figure 4A and B). This is consistent with observed in-phase luminescence patterns for reporter activity of these constructs (Figure 4C). Native Slob transcript also peaked at CT6, while the native $P d p 1$ transcript peaked slightly out of phase with $P d p 1$ 4xCATAC at CT12. The different phases of CLK/CYCregulated $P d p 1$ and $S l o b$ transcripts may reflect subtly different transcriptional activities of their promoters or differing mRNA half-lives $(28,30)$.

\section{CATAC-luc reporters require $c y c$ and per for rhythmicity, but} not induction

Based on the circadian rhythmicity of 4xCATAC-luc reporter genes and the presence of a canonical CACGTG Ebox in the CATAC consensus sequence, we hypothesized that the circadian E-box transcription factor CLK/CYC might be responsible for the observed rhythmic regulation of the CATAC element. To address this, 4xCATAC-luc reporter genes were introduced into a cycle null $\left(c y c^{01}\right)$ genetic background (3). As expected, $P d p 1$ and Slob 4xCATAC lost rhythmicity in the $c y c^{01}$ homozygous background, as did our tim-luciferase positive control (Figure 5, Supplementary Figure S10, Table 3). Multimerized E-box-luc reporters also have been reported to show this in $C l k^{J r k}$ flies (4). However, unlike tim-luc, and multi-E-box-luc constructs, 4xCATAC-luc transgenes did not exhibit a decrease in reporter activity in $c y c^{01}$ homozygotes (Figure 5).

To better understand this result, we performed further analysis of the association between CATAC and observed CLK/CYC binding using published CLK antibody ChIPchip data (38). CATAC elements in the core clock genes, $P d p 1$, tim, per and $c w o$ as well as the clock-controlled gene, Slob, tended to coincide with CLK or PER ChIP signals only in the presence of either an internal canonical E-box or closely-linked external E1E2 motif (Supplementary Figure S11A and B). Moreover, an extended analysis across the entire genome exposed significant enrichment of CLK 
Table 2. Impact of the eyeless mutant $e y a^{2}$ on $4 \times$ CATAC-luc in vivo luminescence rhythms

\begin{tabular}{|c|c|c|c|c|c|c|}
\hline genotype & \#flies & $\% \mathrm{R}$ & $\% \mathrm{WR}$ & $\% \mathrm{AR}$ & $\begin{array}{l}\text { mean } \\
\mathrm{RAE} \pm \mathrm{SEM}\end{array}$ & $\begin{array}{l}\text { mean } \\
\text { period } \pm \text { SEM }\end{array}$ \\
\hline$e y a^{2} /$ CyO; Pdp1 4xCATAC-luc & 18 & 44 & 33 & 22 & $0.67 \pm 0.03$ & $23.03 \pm 0.68$ \\
\hline eya $a^{2} ;$ Pdp1 4xCATAC-luc & 19 & 16 & 26 & 58 & $0.68 \pm 0.06$ & $24.71 \pm 2.28$ \\
\hline eya $a^{2}$ Slob $4 x$ CATAC-luc & 9 & 44 & 44 & 11 & $0.67 \pm 0.05$ & $22.15 \pm 1.17$ \\
\hline
\end{tabular}

Table 3. Impact of the arrhythmic mutant $c y c^{01}$ on $4 x$ CATAC-lucin vivo luminescence rhythms

\begin{tabular}{|c|c|c|c|c|c|c|}
\hline Genotype & \#flies & $\% \mathrm{R}$ & $\% \mathrm{WR}$ & $\% A R$ & Mean RAE \pm SEM & $\begin{array}{l}\text { Mean period } \pm \\
\text { SEM }\end{array}$ \\
\hline Pdp1 4xCATAC-luc; $c y c^{01} /+$ & 7 & 29 & 57 & 14 & $0.70 \pm 0.06$ & $25.74 \pm 1.21$ \\
\hline Pdp1 4xCATAC-luc; cyc $c^{01}$ & 14 & 0 & 14 & 86 & $0.86 \pm 0.09$ & $19.56 \pm 2.59$ \\
\hline Slob $4 x C A T A C-l u c ; c y c^{01}$ & 23 & 4 & 35 & 61 & $0.80 \pm 0.05^{\mathrm{a}}$ & $22.68 \pm 1.98$ \\
\hline Pdp1 mt4xCATAC-luc; cyc $c^{01} /+$ & 22 & 9 & 18 & 82 & $0.73 \pm 0.04^{b}$ & $23.72 \pm 0.41$ \\
\hline Pdp1 mt4xCATAC-luc; $c y c^{01}$ & 18 & 0 & 6 & 94 & $(0.73)$ & $(16.97)$ \\
\hline$p P T l u c ; c y c^{01} /+$ & 24 & 8 & 38 & 54 & $0.81 \pm 0.05^{\mathrm{d}}$ & $23.66 \pm 0.93$ \\
\hline pPTluc; $c y c^{01}$ & 20 & 10 & 25 & 65 & $0.79 \pm 0.08$ & $22.10 \pm 2.19$ \\
\hline tim-luc; cyc $^{01} /+$ & 23 & 83 & 13 & 4 & $0.51 \pm 0.04^{\mathrm{a}, \mathrm{b}, \mathrm{c}, \mathrm{d}, \mathrm{e}}$ & $24.35 \pm 0.46$ \\
\hline tim-luc; $c y c^{01}$ & 23 & 4 & 22 & 74 & $0.82 \pm 0.05^{\mathrm{e}}$ & $19.59 \pm 1.75$ \\
\hline
\end{tabular}

ANOVA with post-hoc Tamhane's T2 $P<0.01^{\mathrm{a}, \mathrm{d}} ; P<0.05^{\mathrm{b}, \mathrm{c}, \mathrm{e}}$.

Table 4. Impact of the arrhythmic mutant $p e r^{01}$ on $4 \mathrm{xCATAC}$-lucin vivo luminescence rhythms

\begin{tabular}{|c|c|c|c|c|c|c|}
\hline Genotype & \#flies & $\% \mathrm{R}$ & $\% \mathrm{WR}$ & $\% \mathrm{AR}$ & Mean RAE \pm SEM & $\begin{array}{l}\text { Mean period } \pm \\
\text { SEM }\end{array}$ \\
\hline Pdp1 4xCATAC-luc; per ${ }^{01} /+$ & 24 & 71 & 25 & 4 & $0.62 \pm 0.03^{\mathrm{a}}$ & $24.92 \pm 0.65$ \\
\hline Pdpl 4xCATAC-luc; per $^{01}$ & 23 & 17 & 13 & 70 & $0.73 \pm 0.05$ & $23.89 \pm 3.00$ \\
\hline Slob $4 x$ CATAC-luc; per ${ }^{01}$ & 22 & 9 & 18 & 73 & $0.76 \pm 0.05$ & $25.18 \pm 2.47$ \\
\hline$P d p 1$ with $S l o b$-like E-boxes $4 x C A T A C$-luc; $p^{01} /+$ & 21 & 29 & 48 & 24 & $0.69 \pm 0.04^{\mathrm{c}}$ & $25.37 \pm 0.77$ \\
\hline$P d p 1$ with Slob-like E-boxes $4 x C A T A C$-luc; per $^{01}$ & 21 & 5 & 5 & 90 & $0.63 \pm 0.17$ & $21.92 \pm 4.19$ \\
\hline Slob mt4xCATAC-luc; per ${ }^{01} /+$ & 24 & 63 & 17 & 21 & $0.59 \pm 0.03^{\mathrm{f}}$ & $23.73 \pm 0.75$ \\
\hline Slob mt4xCATAC-luc; per ${ }^{01}$ & 24 & 0 & 17 & 83 & $0.89 \pm 0.03^{\mathrm{a}, \mathrm{f}, \mathrm{g}, \mathrm{h}}$ & $22.53 \pm 2.67$ \\
\hline pPTluc; per $101 /+$ & 21 & 62 & 24 & 14 & $0.60 \pm 0.04^{\mathrm{h}}$ & $26.75 \pm 0.85$ \\
\hline pPTluc; per $^{01}$ & 21 & 0 & 19 & 81 & $0.85 \pm 0.05^{\mathrm{i}}$ & $20.27 \pm 1.51$ \\
\hline tim-luc; per $101+$ & 24 & 88 & 13 & 0 & $0.46 \pm 0.04^{\mathrm{b}, \mathrm{c}, \mathrm{d}, \mathrm{e}, \mathrm{g}, \mathrm{i}}$ & $23.87 \pm 0.44$ \\
\hline tim-luc; per ${ }^{01}$ & 24 & 17 & 4 & 79 & $0.65 \pm 0.05$ & $18.62 \pm 2.58$ \\
\hline
\end{tabular}

ANOVA with post-hoc Tamhane's T2 $P<0.001^{\mathrm{g}} ; P<0.01^{\mathrm{e}} ; P<0.05^{\mathrm{a}, \mathrm{b}, \mathrm{c}, \mathrm{d}, \mathrm{f}, \mathrm{h}, \mathrm{i}}$.

binding at E1E2 sites, but not CATAC sites (Supplementary Figure S11C; Supplementary Table S2). Taken together, although CATAC-mediated circadian rhythms depended on CLK/CYC, and it is possible that CATAC and CLK/CYCregulated elements act cooperatively in native clock gene promoters, our results indicated that CLK/CYC is not the (only) direct regulator of CATAC. This conclusion is supported by our observation that 4xCATAC-luc differed from multimerized E-box-luc or tim-luc in its ability to maintain high expression levels in the absence of CLK/CYC activity and the fact that in vivo CLK-binding near individual CATAC elements did not appear predictive of their ability to mediate circadian rhythms, but rather of their proximity to canonical E-boxes.
In Drosophila, CLK/CYC is rhythmically repressed by the PER/TIM heterodimer to produce oscillations of clockregulated gene transcripts such as $v r i$ and $P d p l$ (30). A previous study showed that in a $C l k^{r r k}$ or $c y c^{01}$ genetic background tim, per and vri transcription is low (39) - which we confirmed with tim in our bioluminescence assay (Figure 5). However, 4xCATAC does not act in a similar fashion and instead remains at high to intermediate expression levels - a phenotype expected of VRI/PDP1-regulated transcripts such as Clk and cry (30). If CATAC were regulated in a fashion similar to the Clk and cry promoters, we would expect its activity to be not only arrhythmic, but also strongly reduced in a per ${ }^{01}$ background, where increased VRI activity is thought to result in suppression of these promoters. In contrast, CLK/CYC-regulated promoters such 
as tim are expressed at intermediate levels in this background (27) (Figure 6). Although there was some variation among individual wild-type 4xCATAC-luc lines (for example, the Slob 4xCATAC line that was tested showed somewhat reduced expression levels in the $\mathrm{per}^{01}$ background), by and large there was little effect of the $\mathrm{per}^{01}$ mutation on their expression levels (Figure 6). CATAC, therefore, does not appear to be co-regulated with VRI/PDP1 elements.

Additional experiments were performed to examine whether the somewhat variable response of 4xCATACluc expression levels to the per ${ }^{01}$ mutation could be explained by the presence or absence of a canonical E-box. To this aim, we tested our Pdp1 4xCATAC with Slob-like Eboxes (PSE) construct. PSE is a modified version of $P d p 1$ 4xCATAC, which has E-box sequences that were mutated such that their deviation from the CACGTG consensus matched that of the four E-boxes in the Slob 4xCATAC enhancer (Figure 1A). PSE still showed high expression levels in $\mathrm{per}^{01}$ flies, which suggests that the E-box content is not responsible for the observed differences. Nevertheless, in all cases $4 \mathrm{xCATAC}$ lost rhythmicity in the $\mathrm{per}^{01}$ homozygotes, confirming that PER was required for CATAC-mediated rhythms (Figure 6, Supplementary Figure S12, Table 4).

\section{DISCUSSION}

Regulation of circadian gene expression relies on a number of transcriptional elements found in the promoters and introns of core clock and clock-regulated genes. We now know of at least 5 reported functional transcriptional elements that are represented in circadianly regulated genes in Drosophila - the E-box, PERR element, VRI/PDP1-box, CRE element and TER box (27). Among them, the E-box is the best studied. The E-box is a rather versatile regulatory element, capable of functioning with deviations from its canonical form and utilizing flanking sequences and/or nearby E-boxes and regulatory elements to modulate its functionality $(7,8)$.

Noncanonical E-box regulation of circadian genes has previously been identified in Drosophila tim and mammalian $d b p(6,40)$. The CATAC element possesses an Ebox sequence which may be either canonical or noncanonical in nature. Regardless, CATAC is able to generate robust, sustainable rhythms as observed with the $P d p 1$ and Slob 4xCATAC, as well as the $P d p 1$ with Slob-like E-boxes (PSE), constructs. Thus, the considerable variation in quality of E-box sequences between these constructs did not obviously impact reporter gene rhythms. The sequence immediately flanking an E-box element has also been implicated in affecting regulation (9). Simultaneous mutation of residues in the conserved core region of CATAC as well as its E-box, resulted in disrupted rhythms and decreased reporter expression. This suggests that the conserved core sequences are important to CATAC regulation, although it is unclear as to whether this corresponds to rhythmicity, expression levels or both. This question may be addressed in future studies by mutational analyses of only the CATAC core sequence.

As previously observed by McDonald et al. (6), transcriptional elements nearby an E-box can influence circadian gene regulation. The tim promoter has a canonical E-
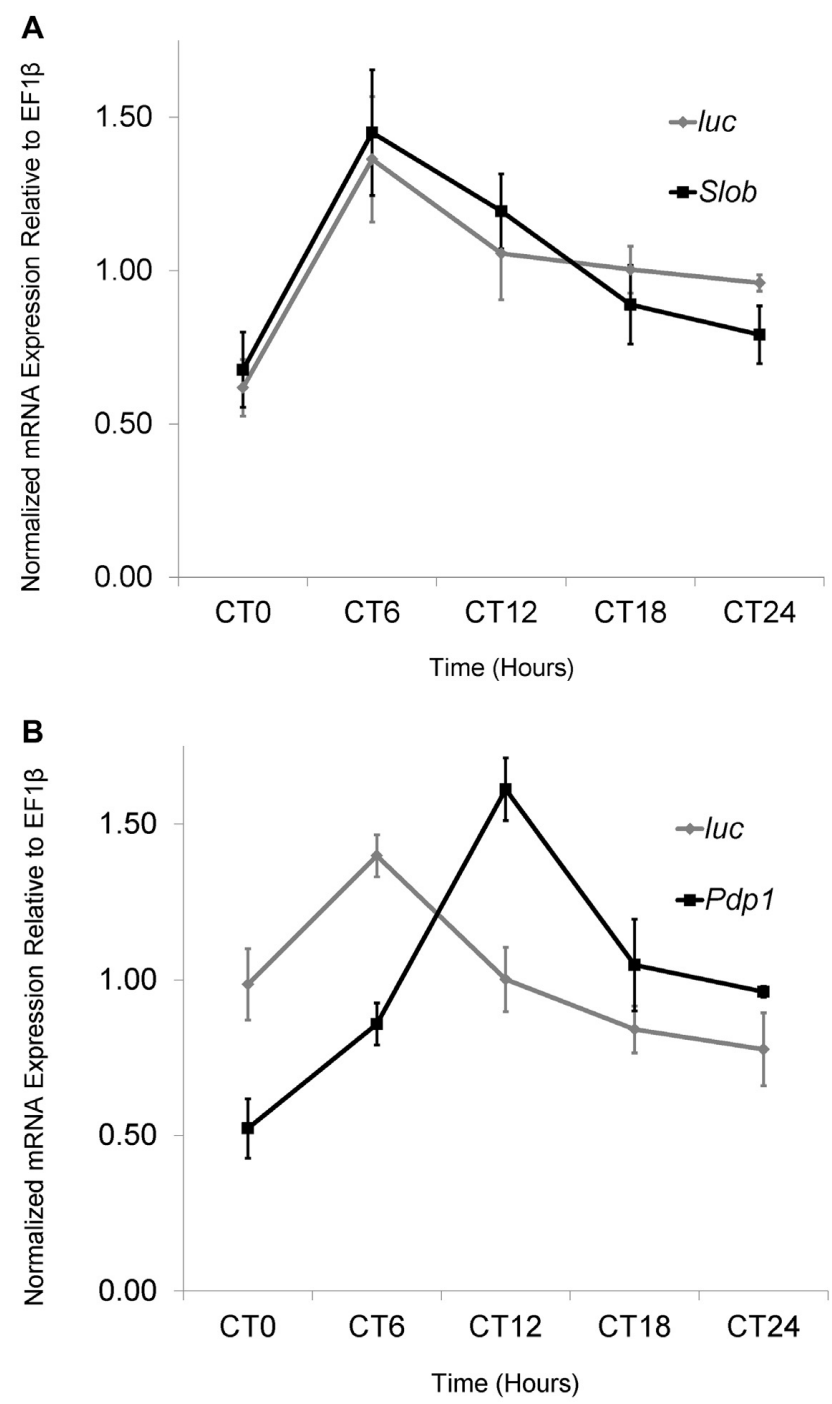

B

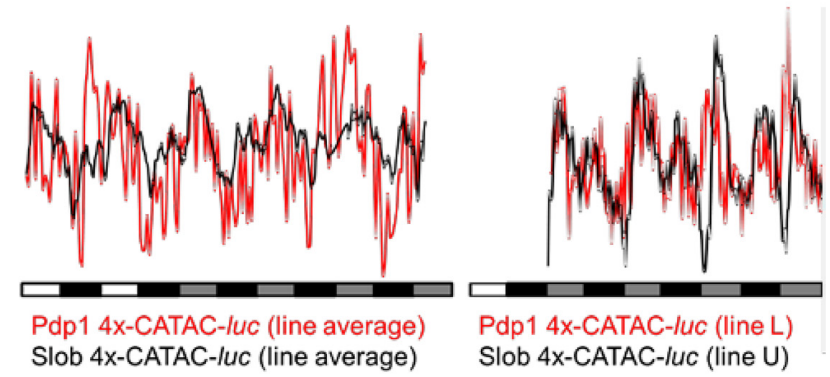

Figure 4. Time course of mRNA expression of $P d p 1$ and Slob 4xCATACluc relative to their respective native gene transcripts in fly heads. (A, B)Transcript profiles were determined relative to $E F 1 \beta$ by qRT-PCR for (A) Slob 4xCATAC-luc (Line U) and (B) Pdp1 4xCATAC-luc (Line L) homozygotes (gray diamonds with gray lines) and compared to the respective native transcripts, $P d p 1$ and Slob (black squares with black lines). Each time point represents the mean data from four independent experiments $( \pm \mathrm{SEM})$. Each profile exhibited significant regulation (Kruskal-Wallis [ $P$-value;peak]: $P d p 1[0.007$; CT12] $P d p 14 x C A T A C$ luc $(0.028 ;$ CT6) Slob $[0.023 ;$ CT6] Slob 4xCATAC-luc $[0.026 ;$ CT6]). (C) The matching mRNA phases for Slob 4xCATAC-luc (A) versus Pdpl $4 x C A T A C$-luc (B) are consistent with in-phase luciferase activity rhythms observed for the across-the-board line average (left; data from Figure 2A) or for the individual lines used in (A) and (B) (right; data from control flies in Figure 5, below). 
4xCATAC Pdp1 (Line L)
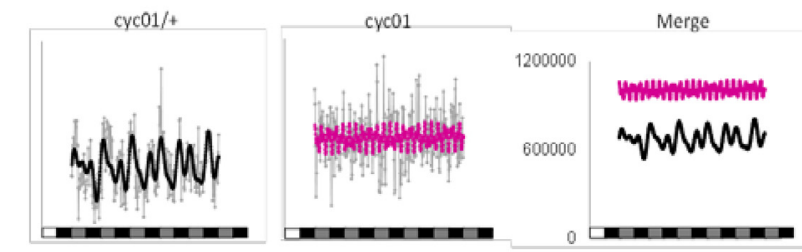

4xCATAC Slob (Line U)
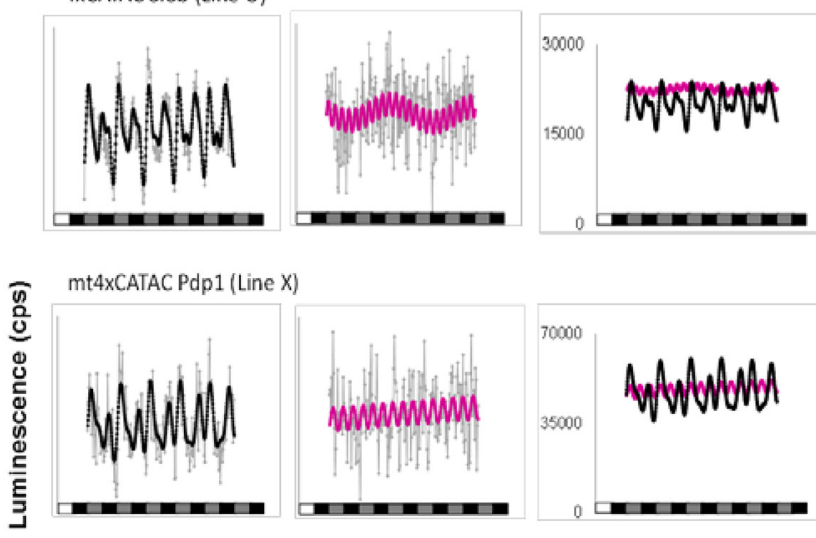

mt4xCATAC Slob (Line S)
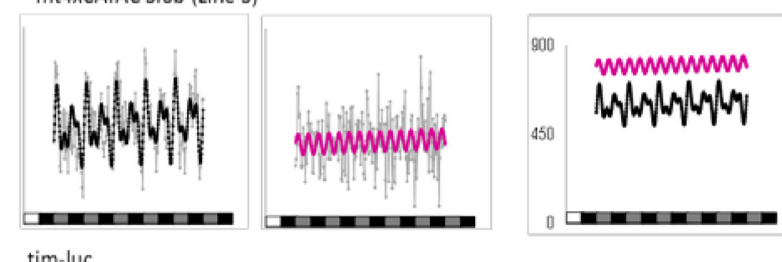

tim-luc
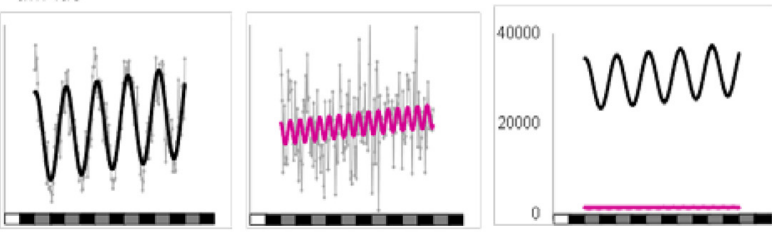

Time (Hours)

Figure 5. Impact of the $c y c^{01}$ mutation on 4 xCATAC-luc rhythmicity and expression. Comparison of detrended, average traces (gray) and their corresponding FFT theoretical overlays (black or magenta) for a single fly line per genotype. Heterozygote controls (black) and the corresponding $c y c^{01}$ homozygotes (magenta) show that without a functioning clock, CATAC fails to yield circadian reporter oscillations. Residual PdpI and Slob mt4xCATAC rhythmicity is further diminished in the homozygous $c y c^{01}$ genetic background. Noticeably, in the $c y c^{01}$ homozygote background tim-luciferase expression levels drop considerably, a phenomenon not observed with $4 \mathrm{xCATAC}$. For statistical measures of the RAE, period and overall rhythmicity refer to Supplementary Figure S10 and Table 3.

box that relies on two proximal cis-regulatory elements that can also interact with CLK/CYC to produce rhythmic transcriptional activity. Genomic analysis of CATAC showed that a high likelihood of CLK/CYC binding coincided with the presence of either a canonical E-box internal to CATAC or the presence of E1E2 motifs in close proximity (Supplementary Figure S11).

For all the similarities that CATAC shares with Ebox motifs, CATAC is not simply another E-box element.

4xCATAC Pdp1 (Line C)

$$
\text { per01/+ }
$$

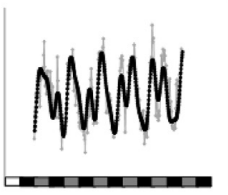

4xCATAC Slob (Line C)
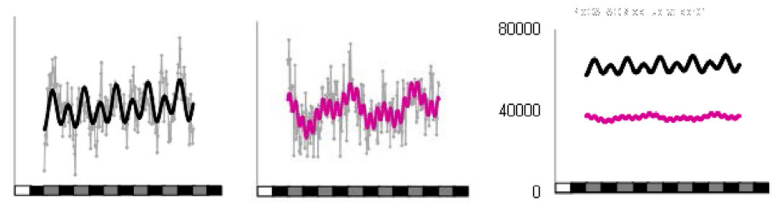

mt4xCATAC Pdp1 (Line B)

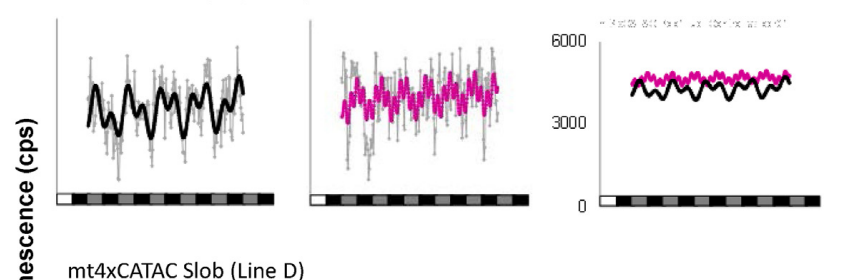

mt4xCATAC Slob (Line D)
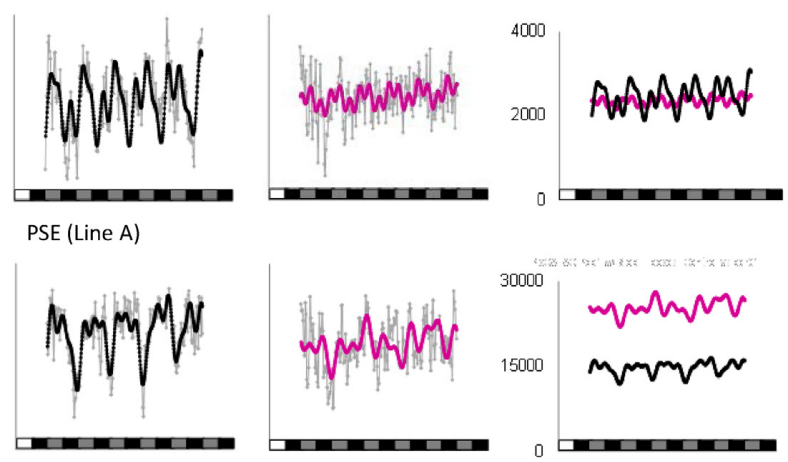

tim-luc
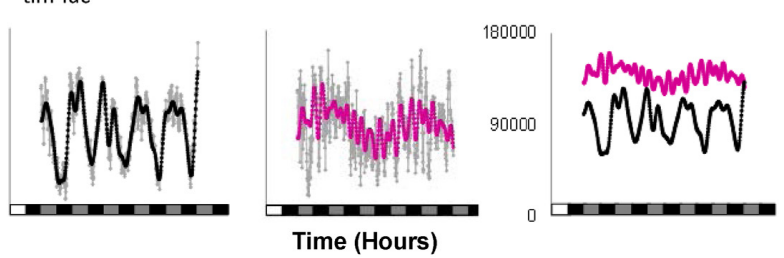

Figure 6. Impact of the per $^{01}$ mutation on 4xCATAC-luc rhythmicity and expression. Comparison of detrended, average traces (gray) and their corresponding FFT theoretical overlays (black or magenta) for a single fly line per genotype. PSE as well as $P d p 1$ and Slob 4xCATAC heterozygote controls (black) and the corresponding per $^{01}$ homozygotes (magenta) show that without a functioning clock, CATAC fails to yield reporter oscillations. Residual Pdp1 and Slob mt4xCATAC rhythmicity is further diminished in the homozygous $\mathrm{per}^{01}$ genetic background. Most reporter constructs, including tim-luc and various CATAC-luc insertions, maintain similar or somewhat increased expression levels in the per $^{01}$ homozygous background; only Slob 4xCATAC expression levels exhibited a mild decrease. For statistical measures of the RAE, period and overall rhythmicity refer to Supplementary Figure S10 and Table 4. 
Normally, an E-box regulated transcriptional element exhibits low reporter expression in a $c y c^{01}$ genetic background and intermediate-to-high expression in per ${ }^{01}(3,41)$. CATAC shows high reporter expression in a $\mathrm{cyc}^{01}$ background and generally intermediate-to-high expression in $\mathrm{per}^{01}$. While CATAC rhythmicity relies on a functioning core clock, CLK/CYC does not appear to be the only potential regulator of CATAC. There is likely another regulator, or regulators, that act on the CATAC to generate increased transcriptional activity in the null backgrounds. Other E-box-binding transcription factors may contribute to the regulation of CATAC along with transcription complexes binding to the conserved core sequence. In particular, the basic helix-loop-helix transcription factor CLOCKWORK ORANGE (CWO) is known to bind E-boxes in clock-controlled genes $(42,43)$ and may, therefore, modulate CATAC activity. However, since CWO exhibits an inhibitory effect on E-box-mediated transcription it is unlikely to be directly responsible for the enhancer activity of CATAC elements.

Thus, we have discovered a novel circadian regulatory element that, although it possesses an E-box-like motif, exhibits non-E-box-like responses. Through use of multimerized CATAC element, we concluded that CATAC is capable of contributing to the rhythmicity of clock genes, such as $P d p 1$ and Slob. Of course, this conclusion comes with the caveat that the multimerized constructs do not represent the complexity of the native genomic environment. Mutational analysis of CATAC sequences in their natural context will constitute an important next step in the functional analysis of this $c i s$-regulatory element. Nevertheless, the identification of CATAC contributes to our knowledge of circadian transcriptional elements, and may be used to further characterize the regulatory regions of clock and clock-regulated genes.

\section{SUPPLEMENTARY DATA}

Supplementary Data are available at NAR Online.

\section{ACKNOWLEDGEMENTS}

The authors would like to thank Mallory Kuchem for assistance with the generation of constructs, Raisa Saab and Chris Uyehara for assistance with some of the genetic and luminescence assays, and Genetic Services, Inc. for assistance with the generation of transgenic flies. We thank the Bloomington Drosophila Stock Center, Drosophila Genomics Resource Center, Michael Young, Michael Rosbash, Jeffrey Hall, Ralf Stanewsky, Liqun Luo and Dan Eberl for the generation or provision of reagents used in this study.

\section{FUNDING}

National Institutes of Health [GM78339 and RR025537 to H.W.]; National Science Foundation [0923366 to H.W.]; Biotechnology and Biological Science Research Council [BB/L023067/1 to H.W.]. Funding for open access charge: RCUK funds held at University of Southampton. Conflict of interest statement. None declared.

\section{REFERENCES}

1. Partch,C.L., Green,C.B. and Takahashi,J.S. (2014) Molecular architecture of the mammalian circadian clock. Trends Cell Biol., 24, 90-99.

2. Allada,R., White,N.E., So,W.V., Hall,J.C. and Rosbash,M. (1998) A mutant Drosophila homolog of mammalian Clock disrupts circadian rhythms and transcription of period and timeless. Cell, 93, 791-804.

3. Rutila,J.E., Suri,V., Le,M., So,W.V., Rosbash,M. and Hall,J.C. (1998) CYCLE is a second bHLH-PAS clock protein essential for circadian rhythmicity and transcription of Drosophila period and timeless. Cell, 93, 805-814.

4. Darlington,T.K., Lyons,L.C., Hardin,P.E. and Kay,S.A. (2000) The period E-box is sufficient to drive circadian oscillation of transcription in vivo. J. Biol. Rhythms, 15, 462-471.

5. Hao,H., Allen,D.L. and Hardin,P.E. (1997) A circadian enhancer mediates PER-dependent mRNA cycling in Drosophila melanogaster. Mol. Cell. Biol., 17, 3687-3693.

6. McDonald,M.J., Rosbash,M. and Emery,P. (2001) Wild-type circadian rhythmicity is dependent on closely spaced $\mathrm{E}$ boxes in the Drosophila timeless promoter. Mol. Cell. Biol., 21, 1207-1217.

7. Kyriacou,C.P. and Rosato,E. (2000) Squaring up the E-box. J. Biol. Rhythms, 15, 483-490.

8. Munoz,E., Brewer,M. and Baler,R. (2002) Circadian transcription. Thinking outside the E-Box. J. Biol. Chem., 277, 36009-36017.

9. Lyons,L.C., Darlington,T.K., Hao,H., Houl,J., Kay,S.A. and Hardin,P.E. (2000) Specific sequences outside the E-box are required for proper per expression and behavioral rescue. J. Biol. Rhythms, 15, 472-482.

10. Adams,M.D., Celniker,S.E., Holt,R.A., Evans,C.A., Gocayne,J.D., Amanatides,P.G., Scherer,S.E., Li,P.W., Hoskins,R.A., Galle,R.F. et al. (2000) The genome sequence of Drosophila melanogaster. Science, 287, 2185-2195.

11. Stapleton,M., Carlson,J., Brokstein,P., Yu,C., Champe,M., George,R., Guarin,H., Kronmiller,B., Pacleb,J., Park,S. et al. (2002) A Drosophila full-length cDNA resource. Genome Biol., 3 , RESEARCH0080.

12. Paquet,E.R., Rey,G. and Naef,F. (2008) Modeling an evolutionary conserved circadian cis-element. PLoS Comput. Biol., 4, e38.

13. Bailey,T.L. and Elkan,C. (1994) Fitting a mixture model by expectation maximization to discover motifs in biopolymers. Proc Int. Conf. Intell. Syst. Mol. Biol., 2, 28-36.

14. Sharma,Y., Cheung,U., Larsen,E.W. and Eberl,D.F. (2002) PPTGAL, a convenient Gal4 P-element vector for testing expression of enhancer fragments in drosophila. Genesis, 34, 115-118.

15. Darlington,T.K., Wager-Smith,K., Ceriani,M.F., Staknis,D., Gekakis,N., Steeves,T.D., Weitz,C.J., Takahashi,J.S. and Kay,S.A. (1998) Closing the circadian loop: CLOCK-induced transcription of its own inhibitors per and tim. Science, 280, 1599-1603.

16. Konopka,R.J. and Benzer,S. (1971) Clock mutants of Drosophila melanogaster. Proc. Natl. Acad. Sci. U.S.A., 68, 2112-2116.

17. Stanewsky,R., Jamison,C.F., Plautz,J.D., Kay,S.A. and Hall,J.C. (1997) Multiple circadian-regulated elements contribute to cycling period gene expression in Drosophila. EMBO J., 16, 5006-5018.

18. Plautz,J.D., Kaneko,M., Hall,J.C. and Kay,S.A. (1997) Independent photoreceptive circadian clocks throughout Drosophila. Science, 278, 1632-1635.

19. Plautz,J.D., Straume,M., Stanewsky,R., Jamison,C.F., Brandes,C., Dowse,H.B., Hall,J.C. and Kay,S.A. (1997) Quantitative analysis of Drosophila period gene transcription in living animals. J. Biol. Rhythms, 12, 204-217.

20. Sullivan,W., Ashburner,M. and Hawley,R.S. (2000) Drosophila Protocols. Cold Spring Harbor Laboratory Press, NY.

21. Sellix,M.T., Currie,J., Menaker,M. and Wijnen,H. (2010) Fluorescence/luminescence circadian imaging of complex tissues at single-cell resolution. J. Biol. Rhythms, 25, 228-232.

22. Goda,T., Mirowska,K., Currie,J., Kim,M.H., Rao,N.V., Bonilla,G. and Wijnen,H. (2011) Adult circadian behavior in Drosophila requires developmental expression of cycle, but not period. PLoS Genet., 7, e1002167.

23. Goda,T., Sharp,B. and Wijnen,H. (2014) Temperature-dependent resetting of the molecular circadian oscillator in Drosophila. Proc. Biol. Sci./R. Soc., 281. 
24. Schmittgen,T.D. and Livak,K.J. (2008) Analyzing real-time PCR data by the comparative C(T) method. Nat. Protoc., 3, 1101-1108.

25. McDonald,M.J. and Rosbash,M. (2001) Microarray analysis and organization of circadian gene expression in Drosophila. Cell, 107, $567-578$.

26. Ceriani,M.F., Hogenesch,J.B., Yanovsky,M., Panda,S., Straume,M. and Kay,S.A. (2002) Genome-wide expression analysis in Drosophila reveals genes controlling circadian behavior. J. Neurosci. Off. J. Soc. Neurosci., 22, 9305-9319.

27. Claridge-Chang,A., Wijnen,H., Naef,F., Boothroyd,C., Rajewsky,N. and Young,M.W. (2001) Circadian regulation of gene expression systems in the Drosophila head. Neuron, 32, 657-671.

28. Wijnen,H., Naef,F., Boothroyd,C., Claridge-Chang,A. and Young,M.W. (2006) Control of daily transcript oscillations in Drosophila by light and the circadian clock. PLoS Genet., 2, e39.

29. Siepel,A., Bejerano,G., Pedersen,J.S., Hinrichs,A.S., Hou,M., Rosenbloom,K., Clawson,H., Spieth,J., Hillier,L.W., Richards,S. et al. (2005) Evolutionarily conserved elements in vertebrate, insect, worm, and yeast genomes. Genome Res., 15, 1034-1050.

30. Cyran,S.A., Buchsbaum,A.M., Reddy,K.L., Lin,M.C., Glossop,N.R., Hardin,P.E., Young,M.W., Storti,R.V. and Blau,J. (2003) vrille, Pdp1, and dClock form a second feedback loop in the Drosophila circadian clock. Cell, 112, 329-341.

31. Zheng,X., Koh,K., Sowcik,M., Smith,C.J., Chen,D., Wu,M.N. and Sehgal,A. (2009) An isoform-specific mutant reveals a role of PDP1 epsilon in the circadian oscillator. J. Neurosci: Off. J. Soc. Neurosci., 29, 10920-10927.

32. Brandes,C., Plautz,J.D., Stanewsky,R., Jamison,C.F., Straume,M., Wood,K.V., Kay,S.A. and Hall,J.C. (1996) Novel features of Drosophila period transcription revealed by real-time luciferase reporting. Neuron, 16, 687-692.

33. Gerlitz,O., Nellen,D., Ottiger,M. and Basler,K. (2002) A screen for genes expressed in Drosophila imaginal discs. Int. J. Dev. Biol., 46, $173-176$.
34. Cheng,Y. and Hardin,P.E. (1998) Drosophila photoreceptors contain an autonomous circadian oscillator that can function without period mRNA cycling. $J$. Neurosci., 18, 741-750.

35. Jaramillo,A.M., Zheng,X., Zhou,Y., Amado,D.A., Sheldon,A., Sehgal,A. and Levitan,I.B. (2004) Pattern of distribution and cycling of SLOB, Slowpoke channel binding protein, in Drosophila. BMC Neuroscience, $\mathbf{5}, 3$.

36. Glossop,N.R. and Hardin,P.E. (2002) Central and peripheral circadian oscillator mechanisms in flies and mammals. J. Cell Sci., 115, 3369-3377.

37. Bonini,N.M., Leiserson,W.M. and Benzer,S. (1993) The eyes absent gene: genetic control of cell survival and differentiation in the developing Drosophila eye. Cell, 72, 379-395.

38. Abruzzi,K.C., Rodriguez,J., Menet,J.S., Desrochers,J., Zadina,A., Luo,W., Tkachev,S. and Rosbash,M. (2011) Drosophila CLOCK target gene characterization: implications for circadian tissue-specific gene expression. Genes Dev., 25, 2374-2386.

39. Blau,J. and Young,M.W. (1999) Cycling vrille expression is required for a functional Drosophila clock. Cell, 99, 661-671.

40. Schibler,U. and Naef,F. (2005) Cellular oscillators: rhythmic gene expression and metabolism. Curr. Opin. Cell Biol., 17, 223-229.

41. Glossop,N.R., Lyons,L.C. and Hardin,P.E. (1999) Interlocked feedback loops within the Drosophila circadian oscillator. Science, 286, 766-768.

42. Kadener,S., Stoleru,D., McDonald,M., Nawathean,P. and Rosbash,M. (2007) Clockwork Orange is a transcriptional repressor and a new Drosophila circadian pacemaker component. Genes Dev. 21, 1675-1686.

43. Matsumoto,A., Ukai-Tadenuma,M., Yamada,R.G., Houl,J., Uno,K.D., Kasukawa,T., Dauwalder,B., Itoh,T.Q., Takahashi,K., Ueda, R. et al. (2007) A functional genomics strategy reveals clockwork orange as a transcriptional regulator in the Drosophila circadian clock. Genes Dev., 21, 1687-1700. 